\title{
BASIC SCIENCE ARTICLE Basal ganglia and thalamic tract connectivity in very preterm and full-term children; associations with 7-year neurodevelopment
}

Deanne K. Thompson (1D ${ }^{1,2,3}$, Wai Yen Loh ${ }^{1,3}$, Alan Connelly ${ }^{3}$, Jeanie L. Y. Cheong ${ }^{1,4,5}$, Alicia J. Spittle ${ }^{1,4,6}$, Jian Chen ${ }^{1,7}$, Claire E. Kelly ${ }^{1}$, Terrie E. Inder ${ }^{1,8}$, Lex W. Doyle ${ }^{1,2,4,5}$ and Peter J. Anderson ${ }^{1,9}$

BACKGROUND: Altered basal ganglia and thalamic connectivity may be critical for cognitive, motor and behavioural impairments common to very preterm ( $<32$ weeks' gestational age) children. This study aims to (1) compare corticostriatal and thalamocortical tract connectivity between very preterm and term-born children at 7 years of age; (2) explore tract connectivity associations with 7-year neurodevelopmental outcomes, and whether these relationships differed between groups.

METHODS: Eighty-three very preterm and 19 term-born ( $\geq 37$ weeks' gestational age) children underwent structural and diffusion magnetic resonance imaging and had a neuropsychological assessment at 7 years. Corticostriatal and thalamocortical tracts were reconstructed and white matter connectivity was estimated with apparent fibre density. RESULTS: Compared with term-born controls, very preterm children had decreased connectivity in tracts linking the caudate to right motor areas $(-10 \%, p=0.03)$ and the thalamus with left motor areas $(-5.7 \%, p=0.03)$. Reduced connectivity in corticostriatal and thalamocortical tracts was associated with adverse motor functioning in both groups $(p=0.06)$. Decreased connectivity of the left caudate and putamen with the lateral prefrontal cortex was associated with lower reading performance for controls $(p=0.06)$.

CONCLUSION: Corticostriatal and thalamocortical tracts are vulnerable to very preterm birth. Poorer connectivity in these tracts may underlie the motor impairments observed in very preterm children.

Pediatric Research (2020) 87:48-56; https://doi.org/10.1038/s41390-019-0546-x

\section{INTRODUCTION}

Children born very preterm (VP) ( $<32$ weeks' gestational age, GA) are at increased risk for brain injury ${ }^{1}$ and poorer cognitive, motor and behavioural functioning. ${ }^{2}$ Understanding the nature of brain abnormalities and how they relate to impaired subsequent functioning is important for informing treatments and interventions aimed at improving outcomes.

The basal ganglia and thalamus are deep grey matter structures of the brain that form a functional network with the cortex. ${ }^{3}$ The cortico-basal ganglia-thalamo-cortical network comprises of segregated circuits that run in parallel to modulate motor, cognitive and affective functions. ${ }^{4}$

The third trimester is a time of active basal ganglia and thalamic development ${ }^{5}$ with the formation of corticostriatal and thalamocortical synapses. ${ }^{6}$ VP birth may delay or alter the normal developmental processes of these deep grey matter structures, including the corticostriatal and thalamocortical tracts, contributing to motor, cognitive and affective functioning deficits which are commonly reported in this population. ${ }^{7}$ Indeed, structural MRI studies have shown that preterm birth is associated with smaller deep grey matter volumes, ${ }^{8-12}$ which are in turn related to poorer cognitive c,11-13 $^{8}$ and motor outcomes. ${ }^{9,11,12,14}$

Few studies have examined the effect of VP birth on the white matter (WM) tracts of the cortico-basal ganglia-thalamo-cortical network. Duerden et al. demonstrated widespread WM microstructure alterations in the WM fibres linking the prefrontal cortex with the striatum of preterm children. ${ }^{15}$ Ball and colleagues highlighted the vulnerability of thalamocortical connectivity in preterm infants and the predictive ability of this WM pathway for cognition at 2 years. ${ }^{16}$ However, to date no study has examined both the corticostriatal and thalamocortical connections of the cortico-basal ganglia-thalamo-cortical network's pathways in the same cohort of VP children, or related these connections to childhood functional outcomes.

Most studies examining WM microstructure in preterm children use measures from the diffusion tensor model. However, tensorderived parameters are voxel averaged measures that are not tract-specific, making them less meaningful in the presence of multiple fibre orientations within a voxel. The constrained

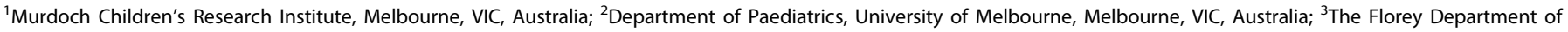

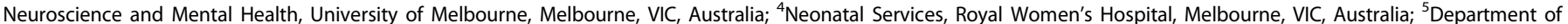

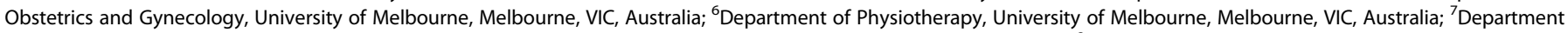

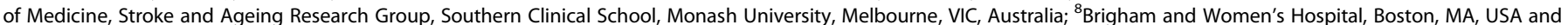
${ }^{9}$ Turner Institute for Brain and Mental Health, School of Psychological Sciences, Monash University, Clayton, VIC, Australia Correspondence: Deanne K. Thompson (deanne.thompson@mcri.edu.au)
}

Received: 1 May 2019 Revised: 14 July 2019 Accepted: 16 August 2019 Published online: 27 August 2019 
spherical deconvolution (CSD) modelling of the diffusion signal is more appropriate than the tensor model for WM tracts with complex architecture such as the corticostriatal and thalamocortical tracts which are in close proximity to other major WM tracts, such as the corona radiata, internal capsule, corticospinal and corticopontine tracts. ${ }^{17}$

Apparent fibre density (AFD) is a fibre bundle specific measure based on the CSD model. ${ }^{18}$ AFD is a measure that is proportional to the intra-axonal volume of each tract within a voxel, and AFD connectivity (sum of AFD throughout a tract divided by tract length) represents the intra-axonal volume per unit area along a tract. The fibre bundle specificity of the AFD measure may result in a more sensitive measure of the underlying biological tissue's structure than tensor-derived measures. ${ }^{18}$

The current study aimed to expand on our current understanding of the effect of VP birth on the cortico-basal gangliathalamo-cortical network by examining both the corticostriatal and thalamocortical tracts as components of the same network using CSD-derived measures of AFD connectivity. In particular, the current study aimed to compare the connectivity of corticostriatal and thalamocortical tracts between VP and term-born children at age 7 years, and explore corticostriatal and thalamocortical tract connectivity associations with neurodevelopmental outcomes (intelligence quotient [IQ], academic achievement, executive functioning, motor functioning, and behaviour) at 7 years of age, and whether these associations differed between the VP and term-born groups. It was hypothesised that compared with termborn children, VP children would have decreased tract connectivity in the corticostriatal and thalamocortical tracts at 7 years, and that decreased tract connectivity in the corticostriatal and thalamocortical tracts would be associated with poorer neurodevelopment in both groups at 7 years.

\section{MATERIALS AND METHODS}

Participants

Participants were part of the Victorian Infant Brain Study (VIBeS), a prospective longitudinal cohort examining brain injury and brain development in VP children. Two hundred and twenty-four VP infants (born $<30$ weeks' GA or $<1250 \mathrm{~g}$ birthweight) without congenital abnormalities, and 46 term-born control infants (born 37-42 weeks' GA and $\geq 2500 \mathrm{~g}$ birthweight) were recruited from the Royal Women's Hospital, Melbourne, Australia between July 2001 and December 2003. At 7 years' corrected age, 197 VP and 43 control participants returned for a follow-up assessment, where
159 VP and 36 control children also underwent brain MRI. The study was approved by the Human Research Ethics Committees of the Royal Women's and Royal Children's Hospitals.

\section{MRI scanning}

Participants were scanned at the Royal Children's Hospital, Melbourne, using a 3 Tesla Siemens MRI scanner. Sequences acquired included $\mathrm{T}_{1}$-weighted structural images $(0.85 \mathrm{~mm}$ sagittal slices, flip angle $=9^{\circ}$, repetition time $=1900 \mathrm{~ms}$, echo time $=2.27$ $\mathrm{ms}$, field of view $=210 \times 210 \mathrm{~mm}$, matrix $=256 \times 256$ ), and singleshot twice-refocused echo-planar diffusion-weighted images with six $b$-value $=0 \mathrm{~s} / \mathrm{mm}^{2}$ images and 45 non-collinear gradient directions with $b$-value $=3000 \mathrm{~s} / \mathrm{mm}^{2}$ (repetition time $=7400 \mathrm{~ms}$, echo time $=106 \mathrm{~ms}$, field of view $=240 \times 240 \mathrm{~mm}$, matrix $=104 \times$ 104 , isotropic voxel size $=2.3 \mathrm{~mm}^{3}$ ).

\section{Segmentation of regions of interest}

All regions of interest (ROIs) were defined on the $T_{1}$-weighted images and then warped into diffusion image space with Advanced Normalisation Registration Tools (ANTs). ${ }^{19}$ Registrations were visually inspected for accuracy.

Segmentation of the basal ganglia (nucleus accumbens, caudate nucleus, and putamen) and thalamus was performed on $\mathrm{T}_{1}$-weighted images using the automated Paediatric Subcortical Segmentation Technique (PSST). ${ }^{20}$ Individual segmentations were checked for accuracy and manually edited by W.Y.L. as required, as previously described. ${ }^{12}$ The caudate nucleus and nucleus accumbens ROls were combined to form a single ROI, termed CNAcc (Fig. 1a).

Cortical reconstruction was performed on the $T_{1}$-weighted images using FreeSurfer (version 4.4), and the cortex was subdivided into 34 regions per hemisphere according to the Desikan-Killiany atlas. FreeSurfer output was inspected and edited according to documentation (https://surfer.nmr.mgh.harvard.edu). For each hemisphere, FreeSurfer cortical labels were combined to form three functional cortical areas: orbital and medial prefrontal cortex (OMPFC), lateral prefrontal cortex (LPFC), and motor area (includes supplementary motor area, premotor cortex, and primary motor cortex; Supp. Table S1).

The prefrontal area was separated from the motor area by the vertical anterior commissure (VAC) line. ${ }^{21}$ The prefrontal area was further divided into lateral and medial portions by making a division along the vertical line on the superior edge of the medial wall of the brain. ${ }^{22}$ To perform the divisions along the VAC line and superior edge of the medial wall, a population template of all

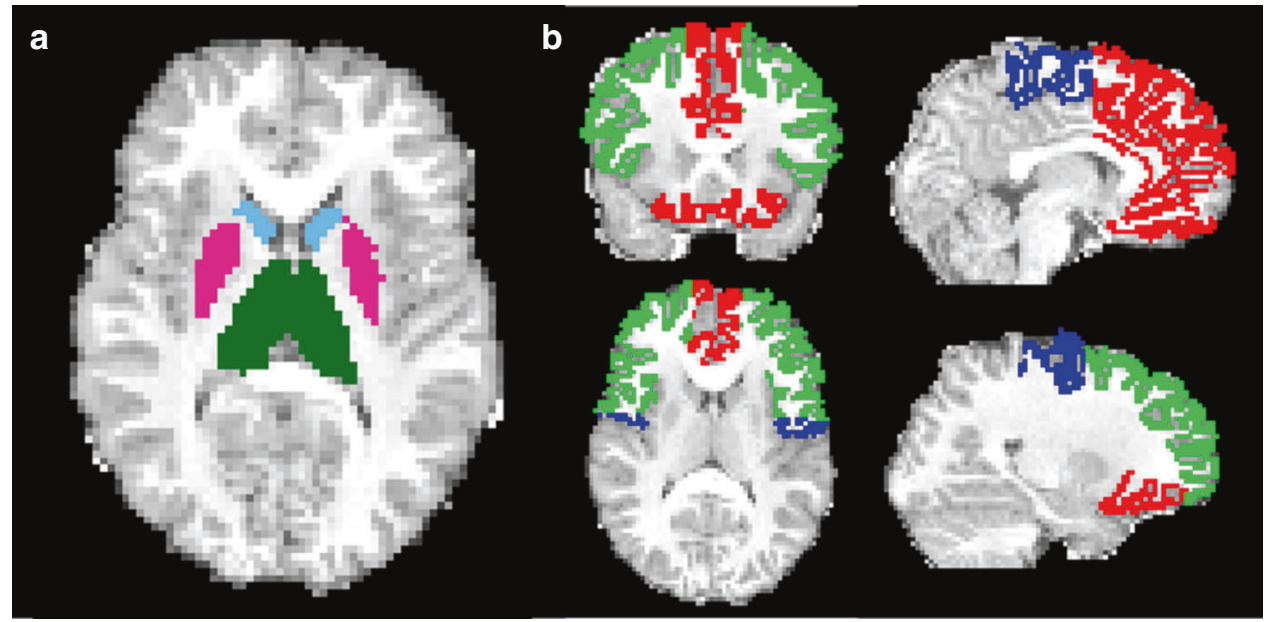

Fig. 1 Regions of interest (ROls) used for tractography of the corticostriatal and thalamocortical tracts for a single term-born participant in diffusion image space. a The basal ganglia and thalamus (seed ROIs) segmented with the Paediatric Subcortical Segmentation Technique. Light blue $=$ caudate nucleus and nucleus accumbens (CNAcc); fuchsia = putamen; thalamus = green. $\mathbf{b}$ Cortical areas (termination ROls) reconstructed with FreeSurfer. Red = orbital and medial prefrontal cortex (OMPFC); green = lateral prefrontal cortex (LPFC); blue = motor area 

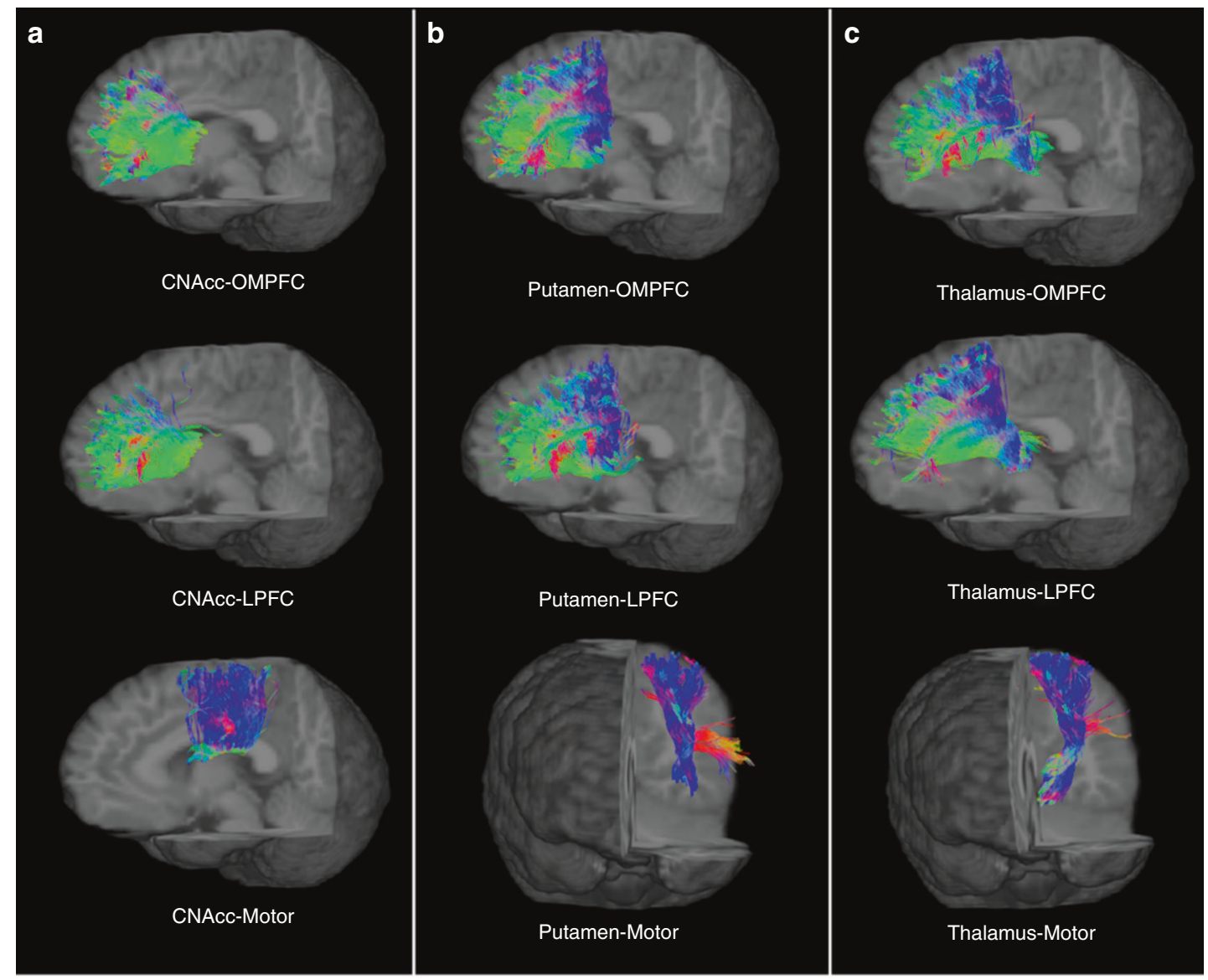

Fig. 2 Corticostriatal $(\mathbf{a}, \mathbf{b})$ and thalamocortical (c) tracts reconstructed in the left hemisphere of a single term-born participant. CNAcc caudate nucleus including nucleus accumbens, OMPFC orbital and medial prefrontal cortex, LPFC lateral prefrontal cortex, green-anteriorposterior, blue-superior-inferior, red-left-right

participants in the current study was constructed from the $\mathrm{T}_{1}$ images using ANTs. ${ }^{19}$ The anterior, posterior, medial, and lateral regions were manually delineated on the template according to the two landmarks (VAC line and superior edge of medial wall) using ITK-SNAP (version 2.2). These demarcated anterior, posterior, medial and lateral regions were warped back from the population template space into each participant's $\mathrm{T}_{1}$ image space to define the OMPFC, LPFC and motor areas over the FreeSurfer cortical labels for each participant (Fig. 1b).

Using MATLAB (version 2010b, The MathWorks, Inc., Massachusetts), additional exclusion ROls were generated in the diffusion image space to prevent the reconstruction of errant streamlines during tractography (Supp. Table S2).

\section{Diffusion-weighted image analysis}

MRtrix software (version 3) was used for pre-processing and analysis of the diffusion-weighted images (http://www.mrtrix.org/). Preprocessing has been detailed elsewhere, ${ }^{18}$ and involved: 1 ) motion correction using a custom method to correct high $b$-value data, involving rigid body registration of each diffusion-weighted volume to a diffusion-weighted mask, incorporating reorientation of the gradient directions; 2) bias field correction by estimating a single multiplicative bias field for each participant using the $b=0 \mathrm{~s} / \mathrm{mm}^{2}$ image and the Local Entropy Minimisation Splines (LEMS) approach, and applying the estimated bias field to correct the intensity of all diffusion-weighted volumes; and 3) intensity normalisation of the diffusion-weighted data. Intensity normalisation was performed to normalise the WM signal in the $b=0 \mathrm{~s} / \mathrm{mm}^{2}$ images across all participants. Fibre orientation distributions (FODs) of the preprocessed diffusion-weighted data were estimated with CSD using an average response function computed from the response functions of all participants $\left(I_{\max }=8\right) .^{23}$

For each hemisphere, 9 corticostriatal and thalamocortical tracts were generated between the 3 basal ganglia and thalamic ROls and the 3 cortical ROls, using the basal ganglia and thalamic ROls as the seed mask, and the cortical ROls as the termination mask. The corticostriatal and thalamocortical tracts for each hemisphere included: caudate and nucleus accumbens-to-lateral prefrontal cortex (CNACC-LPFC), putamen-to-lateral prefrontal cortex (putamen-LPFC), thalamus-to-lateral prefrontal cortex (thalamus-LPFC), caudate and nucleus accumbens-to-motor cortex (CNAcc-motor), putamen-to-motor cortex (putamen-motor), thalamus-to-motor cortex (thalamus-motor), caudate and nucleus accumbens-to-orbital and medial prefrontal cortex (CNAcc-OMPFC), putamen-to-orbital and medial prefrontal cortex (putamen-OMPFC), and thalamus-toorbital and medial prefrontal cortex (thalamus-OMPFC). The exclusion ROls generated with MATLAB, and all other basal ganglia and thalamic ROls that were not used as the seed, were used to restrict streamlines from passing out of the area of the tract. Fibre tracking was done in native space, and in one direction (seed to termination ROI) using the probabilistic algorithm, and 3000 streamlines were generated per tract. The reconstructed corticostriatal and thalamocortical tracts for a single term-born participant are shown in Fig. 2. Tracts were individually inspected for accuracy by W.Y.L. and considered to be realistically reconstructed, even for subjects with brain abnormality in the neonatal period.

The tract connectivity of each tract was estimated using the AFD method via the 'afdconnectivity' command. AFD was measured by firstly calculating the integral of the FOD lobe in a particular direction within a voxel, which is proportional to the 
intra-axonal volume of the tract aligned in that direction. The integrals of all the FOD lobes traversed by the tract of interest were summed to obtain a measure proportional to the total intraaxonal volume, i.e., AFD. ${ }^{18}$ The AFD was divided by tract length to give a measure related to tract connectivity (AFD connectivity).

Neuropsychological assessment

Participants had assessments to evaluate general cognition, executive functioning, academic achievement, motor functioning, and behavioural functioning.

Full-scale IQ was estimated with the Wechsler Abbreviated Scale of Intelligence $(M=100, S D=15)$. Basic academic skills (word reading and math computation) were evaluated with the Wide Range Achievement Test $4(M=100, S D=15)$.

Executive functioning domains were assessed, including attentional control (auditory sustained attention), assessed with the Score! subtest from the Test of Everyday Attention for Children $(M=10, S D=3)$; Working memory, assessed using the Backward Digit Recall from the Working Memory Test Battery for Children $(M=100, S D=15)$; and goal setting, assessed with the Tower of London, and an overall summary score accounting for time to completion and errors was used.

Motor functioning was evaluated using the total score from the Movement Assessment Battery for Children Second Edition (MABC-2), which examines gross motor, fine motor and postural control based upon normative data from the United Kingdom $(M=10, S D=3)$.

General behaviour problems were evaluated using the total behavioural score from the parent report Strengths and Difficulties Questionnaire (SDQ) (maximum score $=50$; higher scores reflect more behavioural problems).

Statistical analyses

Data were analysed using Stata 14.0 (StataCorp, Texas).

Perinatal characteristics were compared between participants and non-participants or between the included VP children and controls using $t$-tests, Mann-Whitney $U$ tests, or $X^{2}$ tests.

Comparing VP AFD connectivity of corticostriatal and thalamocortical tracts at age 7 years with controls (aim 1) was conducted using separate linear regressions for each tract. Estimates were adjusted for age at MRI and sex. Regression coefficient estimates of tract connectivity group differences are presented in the results as a percentage of the mean AFD in the control group.

For Aim 2, the associations between AFD connectivity and 7year neurodevelopmental outcomes in all children in both groups were assessed using separate linear regressions for each tractoutcome combination. A group-by-tract interaction term was included to allow the effect of tract connectivity on outcome measure to vary by group. All estimates were adjusted for age at neurodevelopmental assessment and sex. Regression coefficients are presented in the results as the difference in outcome per $10 \%$ increase in tract connectivity (AFD) for each tract.

All linear regression models were fitted with generalised estimating equations and were reported with robust standard errors to allow for clustering of multiple births. Given the multiple comparisons performed, all $p$-values reported were false discovery rate (FDR) corrected for the 18 tracts investigated using Benjamini and Yekutieli's first method. ${ }^{24}$ FDR correction was applied separately for the group-wise comparison (aim 1) and each of the brain structure-function relationships (aim 2). FDR corrected $p$ values $<0.05$ were considered statistically significant.

\section{RESULTS}

MRI scans

One hundred and fifty-nine VP children and 36 controls underwent MRI at 7 years. One hundred and forty-three VP children and 34 controls had both a $\mathrm{T}_{1}$-weighted and diffusion-weighted dataset acquired. Of these, 83 VP children and 19 controls had data suitable for diffusion analysis. Reasons for exclusion included: (i) diffusion-weighted images acquired using a different sequence whereby gradient directions were non-uniformly distributed on the sphere in $q$-space, which may result in biased estimation of the FODs, ${ }^{25}$ and therefore bias reconstruction and estimation of AFD connectivity (VP, $n=45$; controls, $n=10$ ), (ii) $\mathrm{T}_{1}$ and/or diffusion-weighted images having movement artifact which sometimes resulted in the scan session being aborted early or the image being inadequate to segment ROIs (VP, $n=15$; controls, $n=5)$.

\section{Sample characteristics}

Perinatal characteristics between children from the original cohort with useable MRI data and those without useable MRI data were similar, except VP children included in the study had higher birthweight SD scores $(-0.38$ versus $-0.68, p<0.001)$, a lower proportion of singletons ( $48.2 \%$ versus $63.8 \%, p=0.001)$, and less postnatal steroid exposure $(2.4 \%$ versus $13.5 \%, p<0.001)$.

The proportions of males were similar between the VP and control groups. The VP children and controls differed on the expected perinatal variables, and at 7 years' corrected age, the VP children generally performed more poorly than the controls for the neuropsychological outcomes (Table 1).

\section{VP children versus controls (aim 1)}

Compared with controls, AFD connectivity in the VP group was reduced in the right CNAcc-motor tract [mean difference $-10 \%$ $\left.\left(95 \% \mathrm{Cl}:-16.8 \%,-3.3 \% ; p^{\mathrm{FDR}}=0.03\right)\right]$, and the left thalamus-motor tract [mean difference $\left.-5.7 \%(-9.5 \%,-1.9 \%), p^{\mathrm{FDR}}=0.03\right)$ ]. There was little evidence of AFD connectivity group differences for the other corticostriatal and thalamocortical tracts ( $\left.p^{\mathrm{FDR}}>0.2\right)$ (Fig. 3).

Tract connectivity associations with neurodevelopment (aim 2) For reading (Fig. 4b), there were group interactions for associations with the left CNAcc-LPFC tract $(p=0.003)$ and left putamenmotor tract $(p=0.002)$, where only the controls showed a trend for increased AFD connectivity associated with better reading (left CNACC-LPFC tract, $\beta[95 \% \mathrm{Cl}]$ : $5.3[1.8,8.7] ; p^{\mathrm{FDR}}=0.06$; left putamen-motor tract, $\left.12.4[5.3,19.5], p^{\mathrm{FDR}}=0.06\right)$.

There was a trend for the control group only (group interaction $p=0.003$ ), where increased AFD connectivity in the left putamenLPFC tract was associated with worse working verbal memory $\left(-7.8[-12.6,-2.9], p^{\mathrm{FDR}}=0.06\right.$, Fig. 5b). There was also evidence of a group interaction in the right putamen-LPFC tract $(p=0.004)$ for working verbal memory, but the association between increased AFD connectivity and worse working verbal memory for the controls did not reach statistical significance following FDR correction ( $p^{\mathrm{FDR}}=0.1$ ) (Fig. 5b).

There were weak associations for increased AFD connectivity in many corticostriatal and thalamocortical tracts of VP children and controls with better motor functioning (Fig. 6a). In particular, associations with trends remaining following FDR correction included the putamen-OMPFC tracts (left: $0.7[0.2,1.2], p^{\mathrm{FDR}}=$ 0.06 ; right: $\left.0.7[0.2,1.1], p^{\mathrm{FDR}}=0.06\right)$, left thalamus-OMPFC tract $(0.6$ $\left.[0.2,1.1]), p^{\mathrm{FDR}}=0.06\right)$, right CNAcc-OMPFC tract $\left(0.3[0.1,0.5], p^{\mathrm{FDR}}\right.$ $=0.06$ ), putamen-LPFC tracts (left: $0.6[0.2,1.0], p^{\mathrm{FDR}}=0.06$; right: $0.6[0.2,1.0], p^{\mathrm{FDR}}=0.07$ ), thalamus-LPFC tracts (left: $0.6[0.2,1.0]$, $p^{\mathrm{FDR}}=0.06$; right: $\left.0.7[0.2,1.2], p^{\mathrm{FDR}}=0.06\right)$, right CNAcc-LPFC tract $\left(0.5[0.2,0.9], p^{\mathrm{FDR}}=0.06\right)$, and right CNAcc-motor tract $(0.7[0.2$, $1.1], p^{\mathrm{FDR}}=0.06$ ).

There was little evidence that AFD connectivity in the corticostriatal and thalamocortical tracts was associated with full IQ ( $p^{\mathrm{FDR}}>0.1$ for all tract measures) (Fig. 4a), math computation ( $p^{\mathrm{FDR}}>0.1$ for all tract measures) (Fig. $\left.4 \mathrm{c}\right)$, goal setting $\left(p^{\mathrm{FDR}}>0.3\right.$ for all tract measures) (Fig. $5 c$ ), sustained attention $\left(p^{\mathrm{FDR}}>0.1\right.$ for all tract measures) (Fig. 5a), or behavioural problems ( $p^{\text {FDR }}>0.1$ for all tract measures) (Fig. 6b) following FDR correction. 
Table 1. Perinatal and 7-year characteristics of the study participants

\begin{tabular}{|c|c|c|c|}
\hline Characteristics & $\mathrm{VP}, n=83$ & Control, $n=19$ & $p$ \\
\hline \multicolumn{4}{|l|}{ Perinatal } \\
\hline $\begin{array}{l}\text { Gestational age at birth } \\
\text { (weeks), M (SD) }\end{array}$ & $27.6(1.6)$ & $38.9(1.3)$ & $<0.0005$ \\
\hline Birth weight (g), M (SD) & $1000(208)$ & $3217(542)$ & $<0.0005$ \\
\hline Birth weight SD score, M (SD) & $-0.38(0.85)$ & $-0.05(1.10)$ & 0.16 \\
\hline Singleton, $n(\%)$ & $40(48.2)$ & $17(89.5)$ & 0.001 \\
\hline Male, $n(\%)$ & $37(44.6)$ & $9(47.4)$ & 0.83 \\
\hline $\begin{array}{l}\text { Antenatal corticosteroids, } \\
n(\%)\end{array}$ & $74(89.2)$ & $0(0)$ & $<0.0005$ \\
\hline Postnatal corticosteroids, $n$ (\%) & $2(2.4)$ & $0(0)$ & 0.49 \\
\hline Infection, $n$ (\%) & $28(33.7)$ & $0(0)$ & 0.003 \\
\hline $\begin{array}{l}\text { Oxygen dependency at } \\
36 \text { weeks, } n(\%)\end{array}$ & $24(28.9)$ & $0(0)$ & 0.007 \\
\hline $\begin{array}{l}\text { Cystic periventricular } \\
\text { leukomalacia, } n(\%)\end{array}$ & $2(2.4)$ & $0(0)$ & 0.49 \\
\hline $\begin{array}{l}\text { Intraventricular haemorrhage } \\
\text { grades } 3 / 4, n(\%)\end{array}$ & $3(3.6)$ & $0(0)$ & 0.40 \\
\hline \multicolumn{4}{|l|}{ 7-year } \\
\hline $\begin{array}{l}\text { Age at assessment } \\
\text { (years), M (SD) }\end{array}$ & $7.6(0.3)$ & $7.6(0.2)$ & 0.67 \\
\hline Age at MRI scan (years), M (SD) & $7.6(0.3)$ & $7.6(0.2)$ & 0.56 \\
\hline Full IQ, M (SD) & $99.4(12.3)$ & $106.6(11.7)$ & 0.02 \\
\hline Reading, $M(S D)$ & $102.3(17.0)$ & $109.4(18.5)$ & 0.11 \\
\hline Math computation, M (SD) & $92.8(16.9)$ & $98.2(15.9)$ & 0.21 \\
\hline Sustained attention, M (SD) & $7.7(3.6)$ & $8.5(3.0)$ & 0.40 \\
\hline $\begin{array}{l}\text { Working verbal } \\
\text { memory, M (SD) }\end{array}$ & $87.6(15.7)$ & $99.1(18.3)$ & 0.007 \\
\hline Goal setting, $M(S D)$ & $57.2(12.6)$ & $67.1(10.4)$ & 0.002 \\
\hline Motor function, M (SD) & $9.5(3.2)$ & $11.7(3.1)$ & 0.005 \\
\hline Behavioural problems, M (SD) & $9.1(5.6)$ & $6.6(3.9)$ & 0.09 \\
\hline
\end{tabular}

$V P$ very preterm, $M$ mean, $S D$ standard deviation, $I Q$ intelligence quotient, $M R I$ magnetic resonance imaging

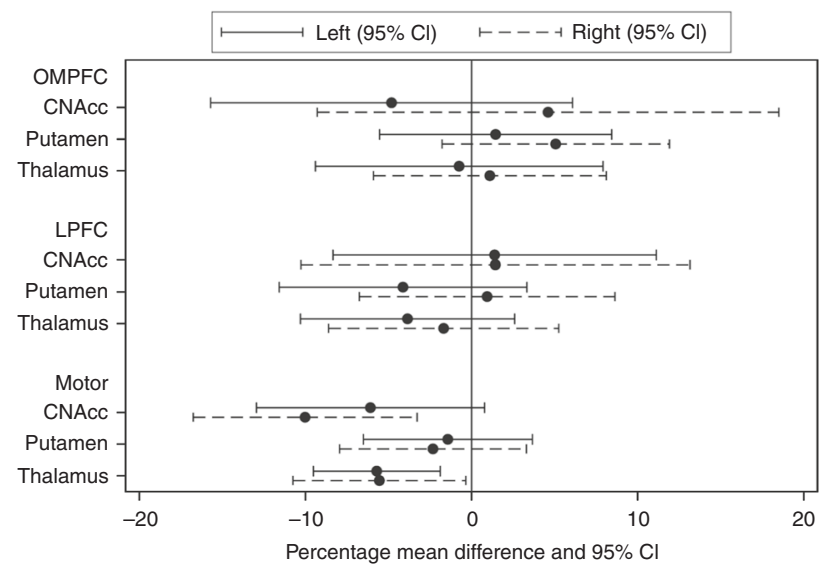

Fig. 3 Corticostriatal and thalamocortical tract connectivity measures in very preterm children compared with controls. $\mathrm{Cl}$ confidence interval, CNAcc caudate nucleus including nucleus accumbens, OMPFC orbital and medial prefrontal cortex, LPFC lateral prefrontal cortex

\section{DISCUSSION}

VP children demonstrated decreased tract connectivity in the right CNAcc-motor tract and left thalamus-motor tract (trend for right) compared with controls. In examining the corticostriatal and thalamocortical tract connectivity relationships with 7-year neurodevelopmental outcomes, increased tract connectivity in multiple basal ganglia and thalamic tracts including the right CNAcc-motor tract were weakly related to better motor development. Some outcome associations were only observed in the controls and not the VP children, notably a weak relationship between increased tract connectivity in the left CNAcc-LPFC and left putamen-LPFC with better reading, and a weak association between increased tract connectivity in the left putamen-LPFC tract with poorer working verbal memory.

VP versus control corticostriatal and thalamocortical tracts

The decreased AFD connectivity in the CNAcc-motor and thalamus-motor tracts of VP children compared with controls indicates a decrease in the space occupied by the axons in the CNAcc-motor and thalamus-motor tracts of VP children compared with controls, which could be a result of decreased axonal density and/or decreased axonal diameter. A possible mechanism that may result in the loss/death of axons, leading to decreased axonal density, is brain injury associated with VP birth. ${ }^{26-28}$ There is also a possibility that axons are underdeveloped following VP birth, resulting in decreased axonal diameter.

Findings from other studies indicate that not only basal ganglia and thalamic axons are affected, but also the neurons within these structures. Smaller basal ganglia and thalamic volumes are reported in those born preterm compared with controls at termequivalent age, ${ }^{9,10}$ childhood, $^{8}$ and adolescence. ${ }^{29}$ In particular, our recent study using the same cohort reported that VP infants had smaller volumes across all basal ganglia nuclei and the thalamus, confirming the specific vulnerability of these structures to VP birth. ${ }^{11}$ This reduction in volume is likely due to dysmaturation via reduced dendritic arborisation, rather than neuronal loss, since neuronal loss is often more associated with severe white matter injury, ${ }^{30}$ of which our cohort had a relatively small incidence. In our longitudinal cohort, we showed that basal ganglia and thalamic growth from infancy to 7 years of age was slower in VP than control children, and the thalamus was still specifically vulnerable in VP children at 7 years of age, meaning there was limited developmental catch-up. ${ }^{12}$ Taking these findings together, the basal ganglia and thalamic macrostructural alterations (smaller volumes) observed at term-equivalent may occur in concert with the microstructural alterations (decreased tract connectivity) observed at 7 years of age, likely initiated by abnormal neuronal growth.

Although the negative impact of VP birth on the CNAcc-motor (right) and thalamus-motor (left) tracts may appear to be hemispheric, the strength and direction of group differences in these tracts had a similar pattern in both hemispheres. Therefore, it seems likely that limited sample size has contributed to the lack of statistical power to detect differences in the contralateral hemisphere. That being said, there is some evidence for hemispheric asymmetry in the basal ganglia and thalamic network. ${ }^{31}$

Contrary to our hypothesis, there was little evidence of group differences in the connectivity of WM tracts linking the prefrontal cortex to the striatum or thalamus. Although we were unable to detect MRI measures that may reflect axonal changes in these tracts as a result of VP birth, we cannot rule out the possibility that there are group differences in myelination that could not be detected. The AFD metric is proportional only to the intra-axonal volume of the WM tract, and does not directly measure myelin. Indeed, dysmaturation of oligodendrocytes that ultimately results in axonal demyelination or hypomyelination has been reported in the preterm brain. $^{26,30}$ 


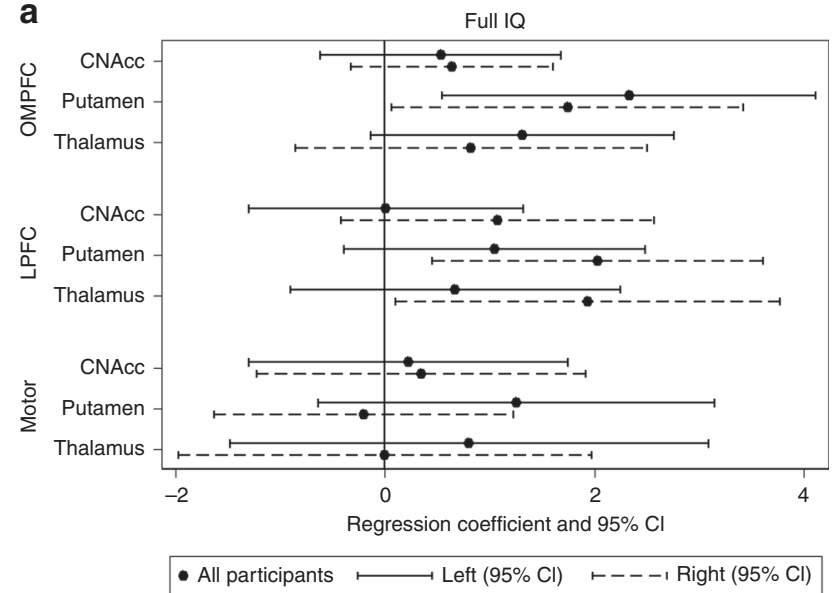

b

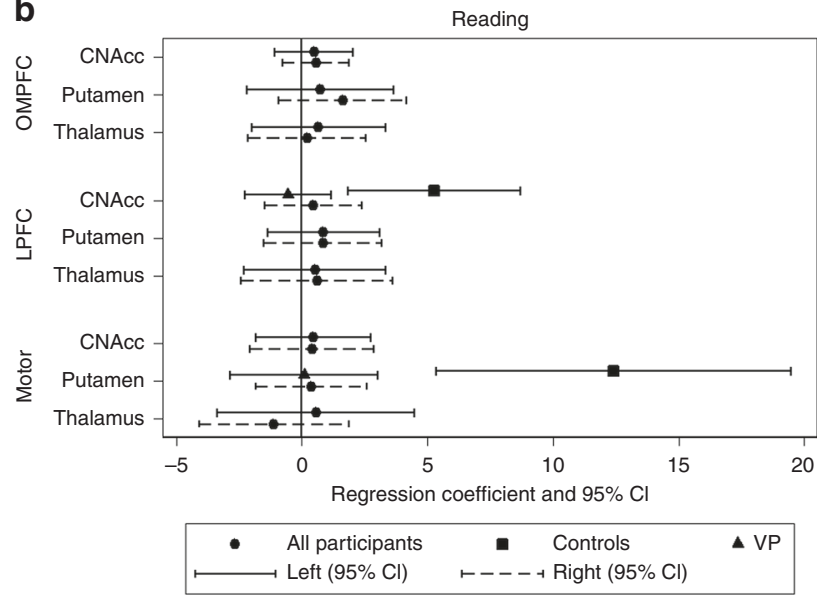

C

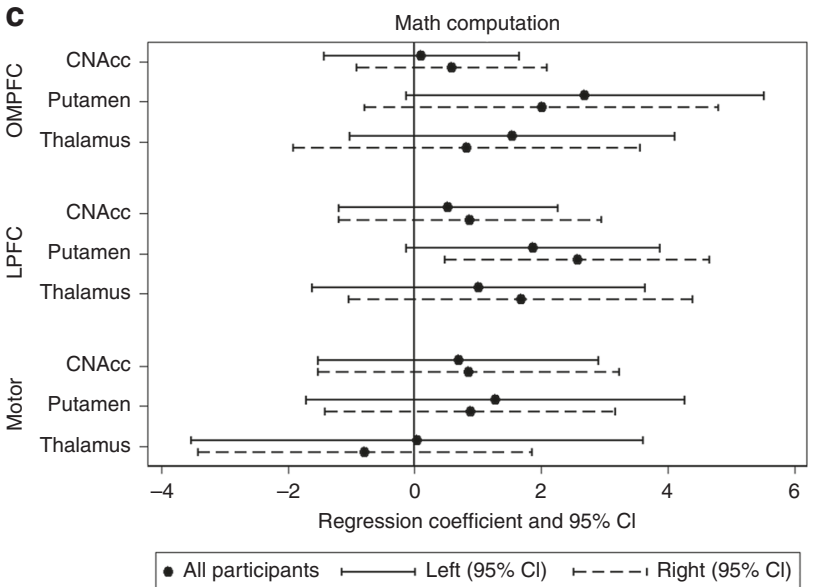

Fig. 4 Corticostriatal and thalamocortical tract connectivity associations with general cognition and academic achievement in all children (very preterm and controls). a Full IQ, b reading, and $\mathbf{c}$ math computation. Cl confidence interval, CNAcc caudate nucleus including nucleus accumbens, OMPFC orbital and medial prefrontal cortex, LPFC lateral prefrontal cortex

Neurodevelopmental outcomes

The strongest association for increased AFD connectivity in putamen, thalamus and CNAcc tracts connecting OMPFC, LPFC, and motor cortical regions was for better motor functioning in VP children and controls at 7 years of age. The association between tracts connecting the caudate nucleus and motor cortex with motor functioning seems intuitive, considering the known involvement of the cortico-basal ganglia-thalamo-cortical circuit in motor functioning. ${ }^{3}$ It is interesting to note that the right CNAcc-motor tract was also found to have reduced connectivity in the VP compared with control comparison in the current study, which would represent a disrupted flow of information between the motor area, and the CNACC and thalamus. The fact that this tract was also associated with motor functioning suggests it is a potential neurological substrate for some of the motor difficulties VP children face. We also found associations between basal ganglia and thalamic volumes in infancy and at 7 years and 7-year motor outcomes in this cohort. ${ }^{11,12}$ This highlights that there are multiple mechanisms for developmental susceptibility of the basal ganglia and thalamus that have significance for motor functioning in those born VP.

At first glance, it may seem unexpected that AFD connectivity between the striatum and prefrontal cortex (OMPFC and LPFC) was related to motor function considering that this cortical area is mostly recruited for cognitive functions. However, there is a known interplay between cognitive and motor processes, where the cortico-basal ganglia-thalamo-cortical network modulates motor function through the learning, planning and execution of movement. ${ }^{32}$ The basal ganglia may also be important for the inhibition of motor responses. ${ }^{33}$ As such, the current study's finding may reflect the roles of these tracts in both motor learning and inhibition of motor responses.

Increased AFD connectivity in the left CNAcc-OMPFC and putamen-motor tracts was associated with better reading in the control group only. This is consistent with findings from a study in healthy school-age students (6-9 years of age) that demonstrated left lateralised functional MRI activation of the basal ganglia (striatum), thalamus and prefrontal cortex to be positively associated with reading speed. ${ }^{34}$ It is interesting that AFD connectivity of the putamen-motor tract was positively associated with reading, a cognitive skill. However, other studies using functional MRI have demonstrated that passive or silent reading of action words activates the motor cortex. ${ }^{35,36}$

There was little evidence that increased AFD connectivity in corticostriatal and thalamocortical tracts was associated with higher IQ in VP children and controls. Contrary to the current study's findings, one study of 20 VP children found WM microstructure (measured with fractional anisotropy) of the corticostriatal tracts was negatively correlated with general cognitive ability (IQ). ${ }^{15}$

There was little evidence that increased AFD connectivity in the corticostriatal or thalamocortical tracts was associated with measures of executive functioning. Unexpectedly, the current study found increased AFD connectivity in the putamen-LPFC tracts was associated with worse working verbal memory in the control group only. Considering that there was a limited sample size ( $n=19$ in the control group), and associations were weak, 

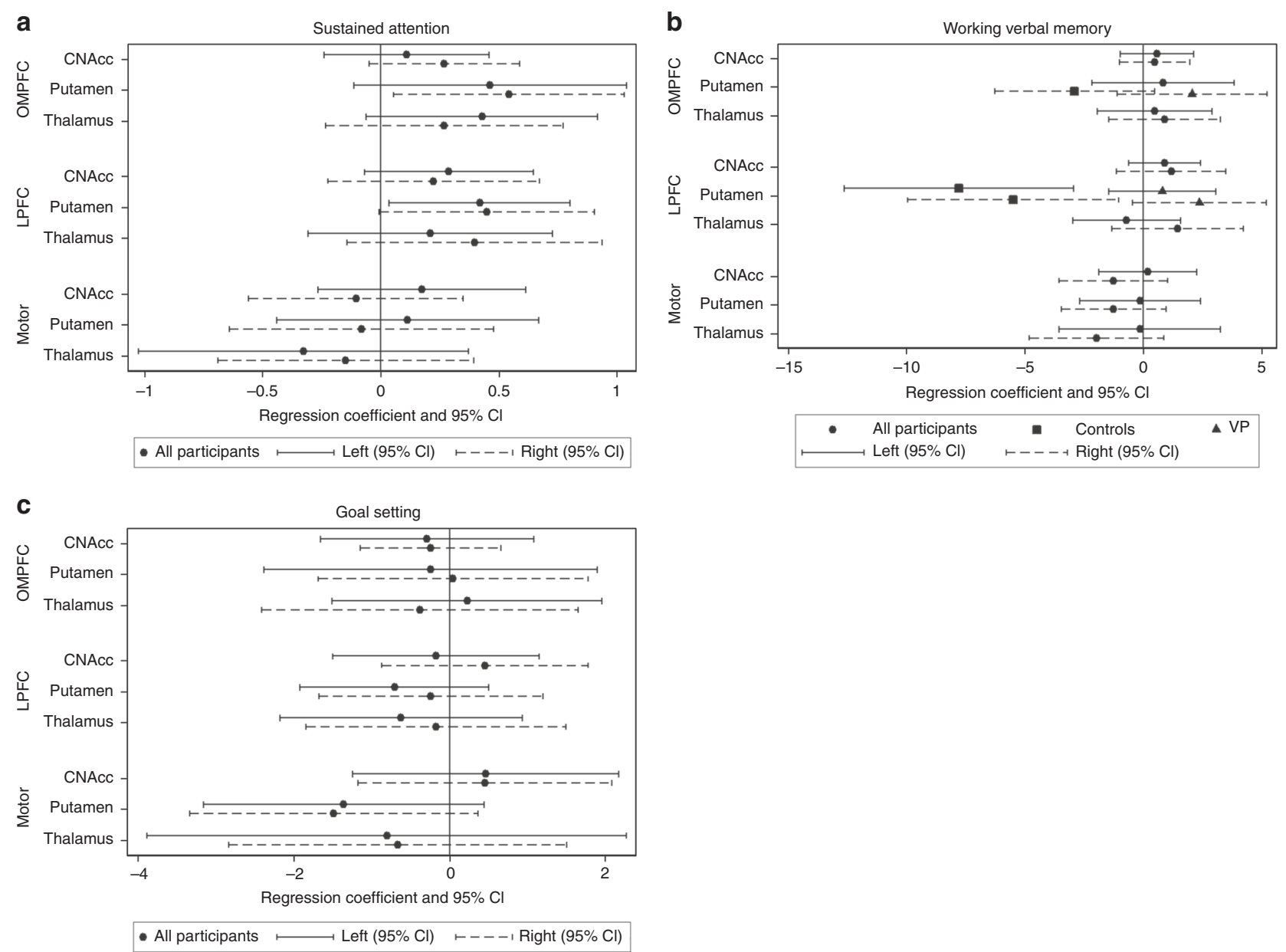

Fig. 5 Corticostriatal and thalamocortical tract connectivity associations with executive functioning in all children (very preterm and controls). a Sustained attention, b working verbal memory, and $\mathbf{c}$ goal setting. Cl confidence interval, CNAcc caudate nucleus including nucleus accumbens, OMPFC orbital and medial prefrontal cortex, LPFC lateral prefrontal cortex

the observed inverse relationship between WM connectivity and working verbal memory in the controls is likely spurious.

There was little evidence that AFD connectivity in the corticostriatal and thalamocortical tracts was associated with behavioural problems. This was contrary to our hypothesis. Indeed, behavioural problems such as apathy, ${ }^{37}$ inattention and impulsivity $^{38}$ and lack of self-control ${ }^{39}$ have been previously associated with various aspects of the basal ganglia and thalamic network. Further research needs to be done to investigate the role of the cortico-basal ganglia-thalamo-cortical network in motivation, impulse control and attentional control within the preterm population.

Interestingly, we have shown that larger infant basal ganglia and thalamus volumes in our cohort were associated with better intelligence and academic achievement, ${ }^{11}$ and these associations were even stronger at 7 years. ${ }^{12}$ Higher growth rates between term-equivalent and 7 years of age were also associated with better IQ, academic achievement and executive functioning and fewer behavioural problems at 7 years, particularly for the CNAcc and pallidum. ${ }^{12}$ Our measures of fibre density showed little association with cognitive functioning, but basal ganglia and thalamic volumes did, indicating the importance of using multiple imaging approaches.

This study together with our previous studies' findings, ${ }^{11,12}$ has identified some of the neural substrates that underlie neurodevelopmental impairments observed in VP children, particularly for motor functioning. Our studies highlight the potential use of basal ganglia and thalamic imaging measures as biomarkers in identifying VP infants who are at risk of poorer neurodevelopment.

Strengths, limitations and future directions

Despite the relatively large cohort, exclusion of data due to inhomogeneous sampling of diffusion gradient directions meant the power to find group differences and associations with outcomes was affected. In particular, the smaller control group relative to the VP group may have meant we could only detect trends that may otherwise have reached statistical significance given a larger sample size, especially in relation to our outcome associations.

While a strength of this study was the use of the AFD measure, an improvement over the tensor model in that it is specific to each fibre population within a voxel, an inherent limitation of the AFD technique is that the amplitudes of the FOD lobes traversed by the tract are added together. ${ }^{18}$ Since the corticostriatal and thalamocortical tracts are adjacent to other major WM tracts, it is possible that a single FOD lobe may contain contributions from more than one tract at some points along their length. Therefore, the measure of AFD connectivity may not be entirely specific to the tracts of interest, which may have masked out some of the expected group differences.

This is the first study to investigate the specific components of the cortico-basal ganglia-thalamo-cortical network. However, it still fails to capture the full complexity of the network. The flow of information within the cortico-basal ganglia-thalamo- 
a

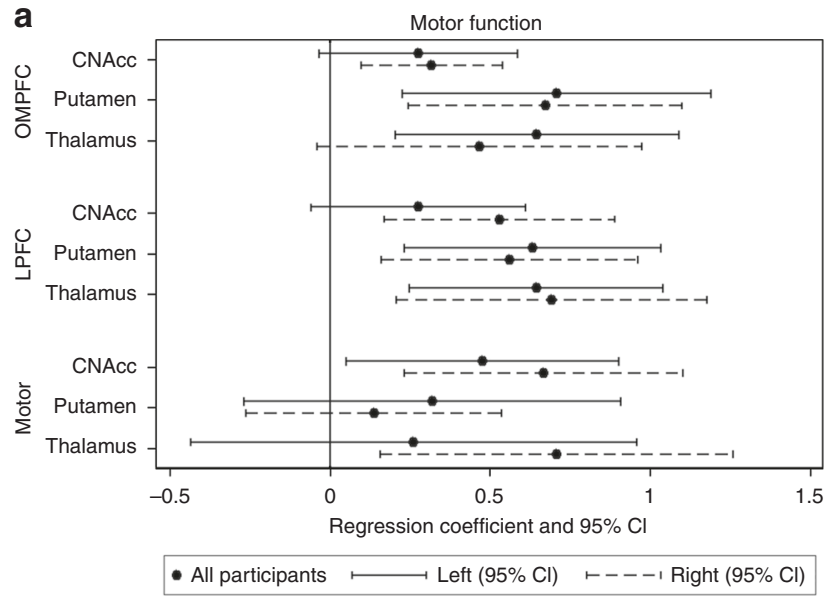

b

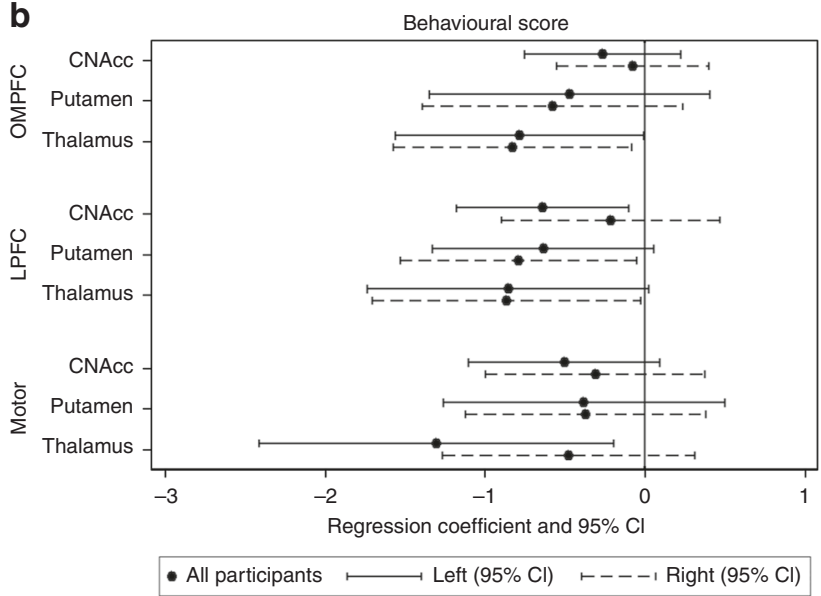

Fig. 6 Corticostriatal and thalamocortical tract connectivity associations with motor and behavioural functioning in all children (very preterm and controls). a Motor function and $\mathbf{b}$ behaviour. $\mathrm{Cl}$ confidence interval, CNAcc caudate nucleus including nucleus accumbens, OMPFC orbital and medial prefrontal cortex, LPFC lateral prefrontal cortex

cortical network is directional, but tractography is unable to discriminate afferent versus efferent pathways. Directionality is especially important in the connections between the cortex and thalamus as both thalamocortical and corticothalamic tracts exist. $^{40}$

Future studies should consider the vulnerability of the corticobasal ganglia-thalamo-cortical network in VP children in relation to the larger whole-brain network. Using connectivity analyses such as graph theory, brain networks can be further characterised into local and global networks and as such, the relationships between the cortico-basal ganglia-thalamo-cortical network and other brain networks can be further explored.

\section{CONCLUSION}

The current study is the first to examine the corticostriatal and thalamocortical tracts as components of the same cortico-basal ganglia-thalamo-cortical network using the advanced diffusion imaging technique, AFD, as a measure of WM microstructure. It confirmed this as an important network affected by VP birth, showing specific vulnerability of the WM tracts linking the motor area with the caudate nucleus (also includes the nucleus accumbens) and thalamus in VP 7-year-old children compared with term-born children, through decreased AFD connectivity. WM connectivity in these tracts was further found to be associated with motor outcome, indicating that altered WM connectivity in these tracts may partially explain the motor deficits observed in VP children.

\section{ACKNOWLEDGEMENTS}

We are grateful for the help and support of the Victorian Infant Brain Studies (VIBeS) and Developmental Imaging groups, as well as the Melbourne Children's MRI Centre at the Murdoch Children's Research institute. Thanks to David Raffelt and Farnoosh Sadhegian at the Brain Research Institute for their assistance with CSD tractography and AFD estimation. We also thank the families and children who participated in this study. This study was supported by Australia's National Health \& Medical Research Council: Centre for Clinical Research Excellence 546519 (L.W.D., P.J.A., T.E.I., and J.L.Y.C.); Centre for Research Excellence 1060733 (L.W.D., P. J.A., J.L.Y.C., D.K.T., A.J.S., and W.Y.L.); Project Grant 491209 (P.J.A., L.W.D., T.E.I., and J.L.Y.C.); Senior Research Fellowship 628371 \& 1081288 (P.J.A.); Career Development Fellowships 1108714 (A.J.S.), 1085754 (D.K.T.), 1141354 (J.L.Y.C.). This study was also supported by the National Institutes of Health (HD058056), the Victorian Government's Operational Infrastructure Support Program, and The Royal Children's Hospital Foundation.

\section{AUTHOR CONTRIBUTIONS}

Each author has met the Pediatric Research authorship requirements as listed below. D.K.T., W.Y.L., A.C., J.L.Y.C., A.J.S., J.C., C.K., T.E.I., L.W.D. and P.J.A. all made substantial contributions to conception and design, acquisition of data, or analysis and interpretation of data; and drafted or revised the article critically for important intellectual content. D.K.T., W.Y.L., J.L.Y.C., A.J.S., J.C., C.K., T.E.I., L.W.D. and P.J.A. gave final approval of the manuscript version to be published.

\section{ADDITIONAL INFORMATION}

The online version of this article (https://doi.org/10.1038/s41390-019-0546-x) contains supplementary material, which is available to authorized users.

Competing interests: The authors declare no competing interests.

Publisher's note Springer Nature remains neutral with regard to jurisdictional claims in published maps and institutional affiliations.

\section{REFERENCES}

1. Volpe, J. J. Brain injury in premature infants: a complex amalgam of destructive and developmental disturbances. Lancet Neurol. 8, 110-124 (2009).

2. Saigal, S. \& Doyle, L. W. An overview of mortality and sequelae of preterm birth from infancy to adulthood. Lancet 371, 261-269 (2008).

3. Nelson, A. B. \& Kreitzer, A. C. Reassessing models of basal ganglia function and dysfunction. Annu Rev. Neurosci. 37, 117-135 (2014).

4. Alexander, G. E., DeLong, M. R. \& Strick, P. L. Parallel organization of functionally segregated circuits linking basal ganglia and cortex. Annu Rev. Neurosci. 9, 357-381 (1986).

5. Makropoulos, A. et al. Regional growth and atlasing of the developing human brain. Neuroimage 125, 456-478 (2016).

6. Kostovic, I. \& Judas, M. The development of the subplate and thalamocortical connections in the human foetal brain. Acta Paediatr. 99, 1119-1127 (2010).

7. Anderson, P. J. Neuropsychological outcomes of children born very preterm. Semin. Fetal Neonatal Med. 19, 90-96 (2014).

8. Peterson, B. S. et al. Regional brain volume abnormalities and long-term cognitive outcome in preterm infants. JAMA 284, 1939-1947 (2000).

9. Inder, T. E., Warfield, S. K., Wang, H., Huppi, P. S. \& Volpe, J. J. Abnormal cerebral structure is present at term in premature infants. Pediatrics 115, 286-294 (2005).

10. Srinivasan, L. et al. Quantification of deep gray matter in preterm infants at termequivalent age using manual volumetry of 3-tesla magnetic resonance images. Pediatrics 119, 759-765 (2007).

11. Loh, W. Y. et al. Neonatal basal ganglia and thalamic volumes: very preterm birth and 7-year neurodevelopmental outcomes. Pediatr. Res. 82, 970-978 (2017).

12. Loh, W. Y., et al. Longitudinal growth of the basal ganglia and thalamus in very preterm children. Brain Imaging Behav. (2019). https://doi.org/10.1007/s11682019-00057-z.

13. Boardman, J. P. et al. A common neonatal image phenotype predicts adverse neurodevelopmental outcome in children born preterm. Neurolmage 52, 409-414 (2010). 
Basal ganglia and thalamic tract connectivity in very preterm and... DK Thompson et al.

14. Setanen, S. et al. Prediction of neuromotor outcome in infants born preterm at 11 years of age using volumetric neonatal magnetic resonance imaging and neurological examinations. Dev. Med. Child Neurol. 58, 721-727 (2016).

15. Duerden, E. G., Card, D., Lax, I. D., Donner, E. J. \& Taylor, M. J. Alterations in frontostriatal pathways in children born very preterm. Dev. Med. Child Neurol. 55, 952-958 (2013)

16. Ball, G. et al. Thalamocortical connectivity predicts cognition in children born preterm. Cereb. Cortex 25, 4310-4318 (2015).

17. Schmahmann, J. D. \& Pandya, D. N. Fiber Pathways of the Brain. (Oxford University Press, New York, 2006).

18. Raffelt, D. et al. Apparent fibre density: a novel measure for the analysis of diffusion-weighted magnetic resonance images. Neuroimage 59, 3976-3994 (2012).

19. Avants, B. B. et al. The optimal template effect in hippocampus studies of diseased populations. Neuroimage 49, 2457-2466 (2010).

20. Loh, W. Y. et al. A new MRI-based pediatric subcortical segmentation technique (PSST). Neuroinformatics 14, 69-81 (2016).

21. Behrens, T. E. J. et al. Non-invasive mapping of connections between human thalamus and cortex using diffusion imaging. Nat. Neurosci. 6, 750-757 (2003).

22. Vijayakumar, N. et al. Thinning of the lateral prefrontal cortex during adolescence predicts emotion regulation in females. Soc. Cogn. Affect Neurosci. 9, 1845-1854 (2014).

23. Tournier, J. D., Calamante, F. \& Connelly, A. Robust determination of the fibre orientation distribution in diffusion MRI: non-negativity constrained superresolved spherical deconvolution. Neuroimage 35, 1459-1472 (2007).

24. Benjamini, Y., Drai, D., Elmer, G., Kafkafi, N. \& Golani, I. Controlling the false discovery rate in behavior genetics research. Behav. Brain Res. 125, 279-284 (2001).

25. Tournier, J. D., Calamante, F., Gadian, D. G. \& Connelly, A. Direct estimation of the fiber orientation density function from diffusion-weighted MRI data using spherical deconvolution. Neuroimage 23, 1176-1185 (2004).

26. Ortinau, C. \& Neil, J. The neuroanatomy of prematurity: normal brain development and the impact of preterm birth. Clin. Anat. 28, 168-183 (2015).
27. Haynes, R. L., Billiards, S. S., Borenstein, N. S., Volpe, J. J. \& Kinney, H. C. Diffuse axonal injury in periventricular leukomalacia as determined by apoptotic marker fractin. Pediatr. Res. 63, 656-661 (2008).

28. Meng, S. Z., Arai, Y., Deguchi, K. \& Takashima, S. Early detection of axonal and neuronal lesions in prenatal-onset periventricular leukomalacia. Brain Dev. 19, 480-484 (1997).

29. Taylor, H. G. et al. Brain volumes in adolescents with very low birth weight: effects on brain structure and associations with neuropsychological outcomes. Dev. Neuropsychol. 36, 96-117 (2011).

30. Back, S. A. Brain injury in the preterm infant: new horizons for pathogenesis and prevention. Pediatr. Neurol. 53, 185-192 (2015)

31. Arsalidou, M., Duerden, E. G. \& Taylor, M. J. The centre of the brain: topographical model of motor, cognitive, affective, and somatosensory functions of the basal ganglia. Hum. Brain Mapp. 34, 3031-3054 (2013).

32. Doyon, J. et al. Contributions of the basal ganglia and functionally related brain structures to motor learning. Behav. Brain Res. 199, 61-75 (2009).

33. Aron, A. R. From reactive to proactive and selective control: developing a richer model for stopping inappropriate responses. Biol. Psychiatry 69, e55-e68 (2011).

34. Alcauter, S. et al. Resting state functional connectivity of the anterior striatum and prefrontal cortex predicts reading performance in school-age children. Brain Lang. 174, 94-102 (2017).

35. Yang, J. \& Shu, H. Passive reading and motor imagery about hand actions and tool-use actions: an fMRI study. Exp. Brain Res. 232, 453-467 (2014).

36. Hauk, O., Johnsrude, I. \& Pulvermuller, F. Somatotopic representation of action words in human motor and premotor cortex. Neuron 41, 301-307 (2004).

37. Walker, F. O. Huntington's disease. Lancet 369, 218-228 (2007).

38. Aron, A. R. et al. Converging evidence for a fronto-basal-ganglia network for inhibitory control of action and cognition. J. Neurosci. 27, 11860-11864 (2007).

39. Hanggi, J. et al. Strength of structural and functional frontostriatal connectivity predicts self-control in the healthy elderly. Front Aging Neurosci. 8, 307 (2016).

40. Redgrave, P. et al. Goal-directed and habitual control in the basal ganglia: implications for Parkinson's disease. Nat. Rev. Neurosci. 11, 760-772 (2010) 